\title{
STUDIES OF PHOTO-EMISSION AND FIELD EMISSION IN AN RF PHOTO-INJECTOR WITH A HIGH QUANTUM EFFICIENCY PHOTO-CATHODE
}

\author{
W. Hartung, ${ }^{*}$ J.-P. Carneiro ${ }^{\dagger}$ D. Edwards, H. Edwards, D. Finley, M. J. Fitch, ${ }^{\ddagger}$ M. Kuchnir, \\ Fermi National Accelerator Laboratory, ${ }^{\S}$ Batavia, Illinois, USA \\ P. Michelato, INFN Milano_-Laboratorio LASA, 20090 Segrate (Milano), Italy \\ D. Sertore, Deutsches Elektronen Synchrotron, Hamburg, Germany
}

\section{INTRODUCTION}

An $18 \mathrm{MeV}$ electron photo-injector facility was constructed at Fermilab in collaboration with UCLA, INFNMilano, University of Rochester, and DESY. The photoinjector is now being operated in partnership with the Northern Illinois Center for Accelerator and Detector Development. The facility consists of an RF gun $[1,2]$ with a cesium telluride $\left(\mathrm{Cs}_{2} \mathrm{Te}\right)$ photo-cathode [3] excited by a pulsed ultra-violet (UV) laser [4], a 9-cell superconducting cavity, a magnetic chicane, and a beam line for experiments with the electron beam. The original goal was to produce a beam with high charge per bunch $(8 \mathrm{nC})$ and low transverse emittance $(<20 \mathrm{~mm}$ mrad) in long bunch trains (800 bunches, $1 \mu$ s apart).

The facility at Fermilab was used to develop, fabricate, and commission an RF gun for the TeSLA Test Facility Linac. This gun has been in use at DESY since November 1998, and its behaviour is under study [5]. A second RF gun was subsequently installed at Fermilab. Recent activities at Fermilab include studies of photo-injector dynamics $[6,7]$ and various experiments with the electron beam: electro-optic sampling of wake fields [8], plasma wakefield acceleration, channeling radiation studies [9], and production of flat beams [10].

Another major goal for the photo-injector was high QE and low field emission ("dark current") from the gun (the latter being a source of unwanted radiation and unwanted background signals). Hence, in between the work outlined above, studies of the photo-emission and field emission behaviour of the gun were carried out. The preliminary results will be summarised in this paper.

\section{PHOTO-INJECTOR DESCRIPTION}

The photo-injector uses UV $(\lambda=263 \mathrm{~nm})$ pulses of duration 10 ps FWHM from the laser. A train of 1 to 10 laser pulses with 0.3 to $1.4 \mu \mathrm{J}$ per pulse was used for the present measurements.

The UV pulses from the laser are directed onto the $\mathrm{Cs}_{2} \mathrm{Te}$ cathode inside the RF gun. The use of $\mathrm{Cs}_{2} \mathrm{Te}$ as a high quantum efficiency (QE, the ratio of emitted photoelectrons to incident UV photons) photo-cathode was first

\footnotetext{
*Present address: NSCL/Cyclotron Lab, Michigan State University.

†Université de Paris XI Orsay, France.

Present address: Institute of Optics, University of Rochester.

${ }^{\S}$ Operated by the Universities Research Association under contract with the U. S. Department of Energy.
}

investigated at CERN [11] and LANL [12], as a compromise between high QE and long life-time. The present system was developed at Milano [13]. The $\mathrm{Cs}_{2} \mathrm{Te}$ film is deposited onto a molybdenum plug via evaporation under ultra-high-vacuum (UHV). Manipulator arms are used to transfer a cathode from the preparation chamber into the $\mathrm{RF}$ gun while remaining in UHV, in order to avoid the deleterious effects of residual gases on the QE. The QE can be recovered in part by "rejuvenating" the cathode with high temperature and UV light $[12,14]$. Two cathodes have been coated and used so far; the present (second) cathode was coated in August 1997 and last rejuvenated in August 1998. Excess Cs was deposited onto this cathode (the deposition time was $75 \%$ longer than for the first cathode). The initial $\mathrm{QE}$ of the second cathode was >10\%; the QE values immediately before and after the last rejuvenation were $0.6 \%$ and 5\%, respectively (DC measurements). Immediately after coating, an increase in QE with time under UV light at room temperature was observed; after rejuvenation, this effect was absent. The coating and early measurements are described in more detail elsewhere [3].

The RF gun is a $1.3 \mathrm{GHz} 1.625$-cell copper cavity, designed for a high duty cycle $(0.8 \%)[1,2]$. The present gun has been in use since March 1999. The measurements described herein were done with $E_{c}=35 \mathrm{MV} / \mathrm{m}\left(E_{c}=\right.$ peak electric field at the cathode), which produces a (total) beam energy of $4.5 \mathrm{MeV}$ at exit of the gun. The RF pulse duration was between 22 and $100 \mu$ s and a $1 \mathrm{~Hz}$ repetition rate was used. The gun is surrounded by 3 focussing solenoids. Under normal conditions, one of the solenoids is used for "bucking," to make $B_{z}$ approximately equal to zero at the cathode. The cathode is placed in the back wall of the gun, with a Be-Cu spring for electrical contact. The pressure in the gun is typically between $10^{-9}$ and $2 \cdot 10^{-9}$ torr.

\section{STUDIES}

The UV energy per pulse and charge per photo-electron bunch were measured with a photo-diode and Integrating Current Transformer (ICT, located $0.38 \mathrm{~m}$ downstream of the gun), respectively, allowing us to infer the QE. The QE was measured on a shot-by-shot basis, selecting one bunch out of the train. Thus the QE includes not only the intrinsic efficiency of the cathode, but also takes into account the transport (or lack thereof) of the photo-electrons from the cathode into the beam tube. The RF phase relative to the laser pulse was typically chosen to be about $90^{\circ}$ (measured from the phase of zero charge extraction), where the ex- 
tracted charge is relatively insensitive to the phase. A large laser spot with low energy per pulse was typically used for the QE measurements, so as to minimise space charge effects on the charge extraction.

The dark current was measured with a Faraday cup inserted into a diagnostic port $0.07 \mathrm{~m}$ after the ICT. The dark current was also measured on a shot-by-shot basis, averaging over a portion of the RF pulse (the dark current values given herein are averaged over the RF period, but not over the RF duty cycle). Thus, the QE and dark current were both recorded at a rate of $1 \mathrm{~Hz}$. Binning and averaging was done later, which improved the signal to noise ratio.

Measurements were done with the usual "round beam" solenoid settings, "flat beam" solenoid settings, and with the solenoids off. The round beam settings correspond to equal current in the primary solenoid $\left(I_{p}\right)$, bucking solenoid $\left(I_{b}\right)$, and secondary solenoid $\left(I_{s}\right): I_{p}=I_{b}=I_{s}=220 \mathrm{~A}$ for the present measurements. These settings make $B_{z}$ close to zero at the cathode. The flat beam settings correspond to zero current in the bucking solenoid and reduced current in the primary and secondary: $I_{p}=170 \mathrm{~A}, I_{s}=70 \mathrm{~A}$, $I_{b}=0$ in the present case, which makes $B_{z}$ about $0.08 \mathrm{~T}$ at the cathode. The maximum $B_{z}$ on axis is about $0.11 \mathrm{~T}$ for both cases. The flat beam settings produce a correlation between the transverse coordinates and momenta, which can be used to make the transverse emittances unequal downstream of the 9-cell cavity via an appropriate set of magnetic elements [10], hence the name. Note that the flat and round beam settings produce the same charge distribution in the electron bunch as it is accelerated through the gun; only the correlations are different.

\section{RESULTS}

After having the RF off for a shift or longer, we observe a slow increase with time in both the QE and the dark current after turning on the RF and solenoids with round beam settings; eventually a steady-state value is reached. Some examples are shown in Figure 1. In Figure 1a, the initial dark current is about $70 \mu \mathrm{A}$, and a steady state value about 8 times higher is reached after many hours. In the case of the $30 \mu$ sF pulse, the RF field was drifting up (as high as $36.5 \mathrm{MV} / \mathrm{m}$ ) and down (as low as $34.5 \mathrm{MV} / \mathrm{m}$ ) relative to the nominal value. The exponential dependence of the field emission current on the electric field serves to amplify the fluctuations, which accounts for the relatively large fluctuations in the dark current. In the case of the $100 \mu \mathrm{s}$ RF pulse, the field level was more stable. After about $700 \mathrm{~min}-$ utes, the RF was turned off for about 30 minutes. When the RF was turned back on, the dark current was lower than it had been just before the RF was turned off. As can be seen, the rate of change and the steady state value of the dark current are insensitive to RF pulse length.

Figure $1 \mathrm{~b}$ indicates that the $\mathrm{QE}$ follows the same general pattern as the dark current (note the different time span). In one case, the initial QE is $0.3 \%$, increasing to $0.8 \%$ after about 7 hours. In the other case, the QE increases by a factor of 2 in 5 hours. In both cases, the initial rate of increase
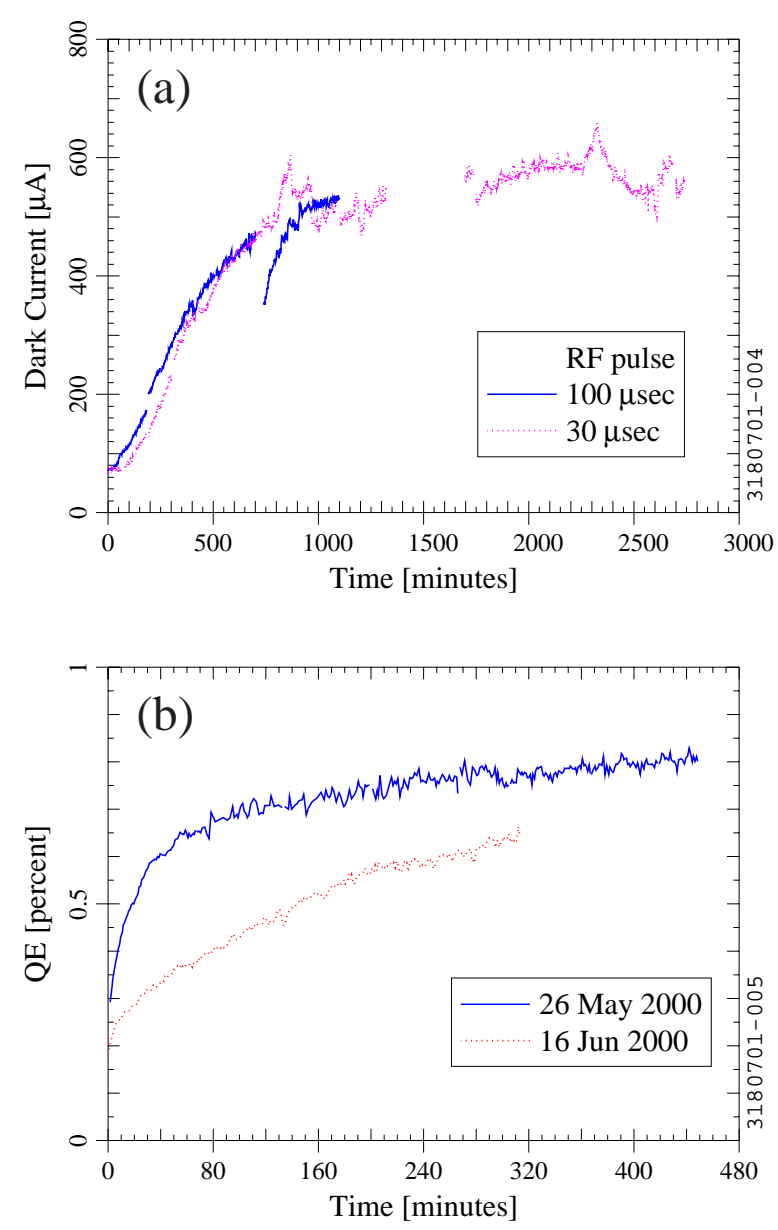

Figure 1. Measurements of (a) dark current and (b) quantum efficiency as a function of time with round beam solenoid settings. A $22 \mu$ s RF pulse was used for (b).

in QE is rapid, and then becomes more gradual, which is in contrast to the dark current's behaviour. After running for extended periods, a QE of about $2 \%$ is typical.

If we turn off the solenoids after running with round beam settings for several hours, we observe a gradual decrease in $\mathrm{QE}$ and dark current with time. Likewise, the dark current and QE decrease with time if we switch to flat beam settings. The effect is illustrated by switching between solenoid settings periodically, producing a "zig-zag" pattern in the QE and dark current. Examples are shown in Figure 2; another example is shown in [10]. Initially, the dark current and QE are relatively constant, having reached a steady state value. Upon changing the settings, the QE and dark current begin to vary with time. Steady state values are not reached after keeping the same settings for an hour. (After running with flat beam settings for several hours, the dark current and QE might reach $140 \mu \mathrm{A}$ and $0.4 \%$ or so, respectively.)

Although it is typical to reach a maximum dark current of $600 \mu \mathrm{A}$ or so (Figure 1a), significantly higher dark current has been observed. Dark current values in excess of $1 \mathrm{~mA}$ are not unusual; the highest dark current observed so far is about $3 \mathrm{~mA}$ (at $E_{c}=35 \mathrm{MV} / \mathrm{m}$ ). The circumstances 

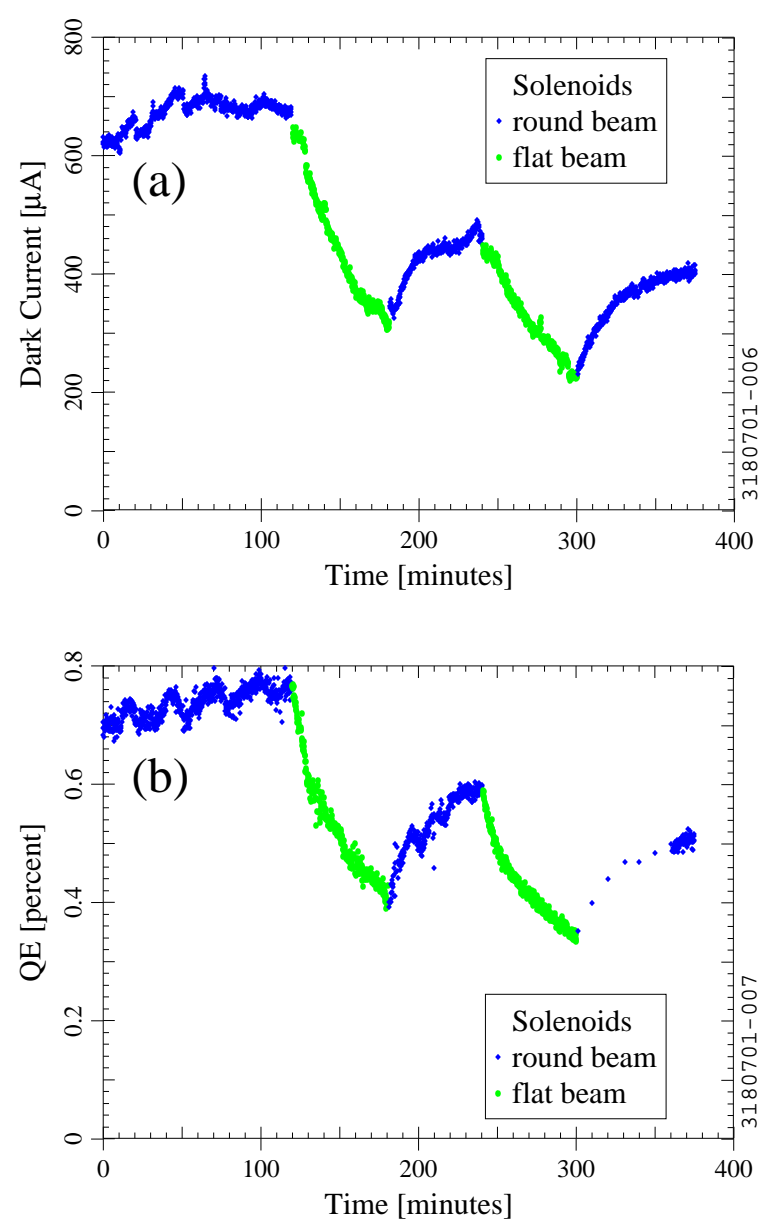

Figure 2. Measurements of (a) dark current and (b) QE as a function of time, switching between round and flat beam solenoid settings. The RF pulse length was $80 \mu \mathrm{s}$.

which produce this much higher dark current have not yet been understood.

The QE and dark current were measured simultaneously in Figure 2. Starting at $t=300$ minutes, the laser shutter was kept closed most of the time. The shutter was opened for 10 shots every 12 minutes to check the QE, so that the average laser intensity was not zero, but was reduced by more than a factor of 70 relative to the usual conditions. As can be seen, the QE (and dark current) increased with the round beam settings, whether the laser shutter was always open or mostly closed. This suggests that the UV light from the laser is not the main cause of the increase in the QE (and dark current). Thus the mechanism is different from the rejuvenation effect that is observed with the combination of high temperature and UV light.

The $\mathrm{Cs}_{2}$ Te cathode was replaced with an uncoated cathode (i.e. a bare Mo plug) for 1 shift. The dark current with the Mo cathode was about $100 \mu \mathrm{A}$, and it did not change significantly over 5 hours of operation with round beam settings or with another 2 hours of operation with flat beam settings. When the coated cathode was returned to the gun, the dark current increased slowly with time, as observed previously. This suggests that the variation in dark current is due to the $\mathrm{Cs}_{2} \mathrm{Te}$ film, not due to effects occurring in the gap between the cathode and the wall of the gun, at the $\mathrm{Be}-\mathrm{Cu}$ spring, or elsewhere inside the gun.

The photo-current and dark current were imaged on a flag downstream of the chicane. A dozen or more distinct spots or "streaks" of dark current were seen, each in the shape of a comet or crescent moon. The distorted shape is due to electrons being emitted at different RF phases, being accelerated to different energies, and being guided to differently by the magnetic elements. To get an idea of the location of the sources of dark current, the laser spot was steered to different transverse positions on the photocathode. At each position, the RF phase was varied in order to trace out the shape of a dark current streak at that location. This allowed us to get an idea of which dark current spots originated on the photo-cathode; more than half of the sources appeared to be on the photo-cathode itself.

\section{CONCLUSION}

A time dependence is observed in the photo-emission and field emission from the photo-injector at Fermilab. The focussing solenoids can be adjusted to cause the QE and dark current to increase or decrease with time. The observed variation is in excess of a factor of 5 in the QE and a factor of 10 in the dark current, with time constants measured in hours. The evidence suggests that the source of the time-dependent behaviour is the $\mathrm{Cs}_{2} \mathrm{Te}$ cathode. The time dependence in the QE (and dark current) occurs even in the absence of UV light. Under favourable circumstances, the $\mathrm{QE}$ is about $2 \%$, even though it has been almost 4 years since the cathode was coated. The mechanism which produces the variation in photo-emission and field emission is not yet understood, and all of the relevant variables have probably not been identified.

\section{REFERENCES}

[1] E. Colby, in Proceedings of the XIX International Linac Conference, Report ANL-98/28, 1998, p. 758-762.

[2] J.-P. Carneiro et al., in Proceedings of the 1999 Particle Accelerator Conference, 1999, p. 2027-2029.

[3] A. Fry et al., in Proceedings of the XIX International Linac Conference, Report ALN-98/28, 1998, p. 642-644.

[4] A. R. Fry et al., Nucl. Instrum. Methods Phys. Res. A430, p. 180-188 (1999).

[5] D. Sertore et al., presented at the 22nd International Free Electron Laser Conference (August 2000).

[6] J.-P. Carneiro et al., in Proceedings of the XX International Linac Conference, Report SLAC-R-561, 2000, p. 104-106.

[7] M. J. Fitch et al., Ibid., p. 131-133.

[8] M. J. Fitch et al., Phys. Rev. Lett. 87034801 (2001).

[9] R. A. Carrigan, Jr. et al., Fermilab-Conf-01/029-E (March 2001)

[10] Don Edwards et al., these proceedings.

[11] E. Chevallay et al., Nucl. Instrum. Methods Phys. Res. A340, p. 146-156 (1994).

[12] S. H. Kong et al., Ibid. A358, p. 276-279 (1995).

[13] P. Michelato et al., in Proceedings of the Fifth European Particle Accelerator Conference, 1996, p. 1510-1512.

[14] A. di Bona et al., J. Appl. Phys. 80, p. 3024-3030 (1996). 